Review

\title{
Progress of Luminescent Carbon Dots in Biomedicine Engineering
}

\author{
Guangxia Shen ${ }^{1,2}$, Meixin $\mathrm{Hu}^{1,2}$, Jianrong $\mathrm{Qi}^{1,2}$ \\ ${ }^{1}$ Department of Instrument Science and Engineering, School of Electronic Information and Electrical Engineering, Shanghai Jiao \\ Tong University, Shanghai 200240, China. \\ ${ }^{2}$ Shanghai Engineering Research Center for Intelligent diagnosis and treatment instrument, Shanghai 200240, China. \\ Corresponding author. E-mail: gxshen@sjtu.edu.cn
}

Received: Sep. 18, 2016; Accepted: Sep. 20, 2016; Published: Sep. 29, 2016.

Citation: Guangxia Shen, Meixin Hu and Jianrong Qi, Progress of Luminescent Carbon Dots in Biomedicine Engineering. Nano Biomed. Eng., 2016, 8(3): 190-202.

DOI: 10.5101/nbe.v8i3.p190-202.

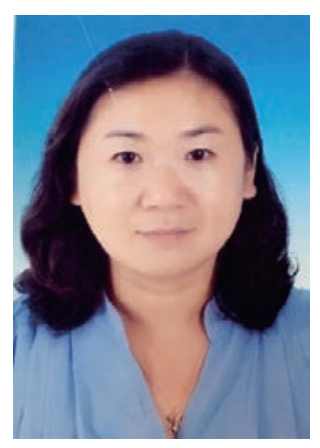

Guangxia Shen obtained his Ph.D. degree in Physical Chemistry from Xiamen University in 2004. She then worked as a postdoctoral in Shanghai Institute of Applied Physics, Chinese Academy of Science from 2005 to 2007. She joined the Research Institute of Micro/Nano Science and Technology at Shanghai Jiao Tong University in 2007, and is now an Associate Professor for materials science and engineering. Dr Shen's research interests cover the controllable fabrication of Carbon nanomaterials by CVD/Microwave/Electrochemical technologies, nanofabrication and nanomanipulation via Atomic Force Microscopy. She has published more than 20 peer-viewed papers in international journals and holds 10 patents. She has presided two National Science Fund projects and one 863 plan.

\begin{abstract}
Carbon nanodots (C-dots), as a new emerging star, have attracted great attention in recent years. Its unique properties have been developed and applied to energy conversion/storage, bioimaging, drug delivery, sensor and other biological related aspects. In this review, we introduce various synthetic methods, basic photoluminscene properties, PL mechanism and then focus on the most recent progress in targeted drug and gene delivery guided by multimode bioimaging technologies in cancer therapy. We also speculate on an outlook towards future developments for their use in bioimaging, drug delivery, sensors, diagnostics and composites.
\end{abstract}

Keywords: Carbon dots; Bioimaging; Biosecurity

\section{Introduction}

As a new family of carbonaceous nanomaterials, carbon dots (C-dots) have drawn increasing attention since they were first obtained in 2004 [1]. Compared with organic dyes and semiconductor quantum dots (QD), C-dots have their unique properties such as excellent water dispersibility, chemical stability and photostability, ease of functionalization, excitationdependent multicolor emission, good cell permeability, and so on. Most importantly, due to their nontoxic and biocompatible properties, C-dots are considered to be a potential alternative to semiconductor quantum dots in biological medicine [2, 3]. Moreover, researchers 
recently found that C-dots exhibit strong luminescence upon two-photon excitation in the near infrared (NIR) region, which further expands their applications in bioimaging [4]. In this review, we summarize the synthetic methods, basic photoluminscene (PL) properties, PL mechanism of C-dots and then focus on the most recent progress in targeted drug and gene delivery guided by multimode bioimaging technologies. Meanwhile, we also speculate on an outlook towards future development of biological medicine base on our research experience about C-dots for ten years.

\section{Synthetic Methods of C-Dots}

Many synthetic routes of C-dots have already been reported since discovered and subsequently dented as carbon nanodots. All in all, the preparation methods can be classified into two main groups: top-down and bottom-up methods (Scheme 1). Top-down methods consist of arc discharge [1, 5], laser ablation [6-9], and electrochemical oxidation [10-13], where the C-dots are formed or "broken off” from a larger carbon structure (such as graphite, carbon rods, graphene Oxides and carbon nanotubes). Bottom-up strategies consist, for example, of combustion/hydrothermal/ acidic oxidation/ultrasonic [14-16], supported synthetic $[17,18]$, or microwave methods [19-22] during which the C-dots are formed from molecular precursors. Meanwhile, the nuance of reaction parameters during synthesis can tremendously affect on the properties of the C-dots, various carbon precursor and synthetic methods. In particular, functional groups such as carboxyl, amino, carbonyl, hydroxyl, and other groups on C-dots facilitate further modifications, which expand the range and heighten the sensitivity and selectivity of the applications.

Sun and co-workers were one of the earliest groups reported researches on C-dots [23, 24]. In 2006, they have first purpose fully produced C-dots by laser ablation. The quasi-spherical C-dots particles with 3 to $10 \mathrm{~nm}$ in size were obtained through a series of complicated procedures including hot-pressing a mixture of graphite powder and cement into graphite target, laser ablating graphite target in a flow of argon gas at $900{ }^{\circ} \mathrm{C}$ and $75 \mathrm{kPa}$, hydrothermal heating by concentrate $\mathrm{HNO}_{3}$, surface passivation by diamineterminated poly(ethylene glycol) $\left(\mathrm{PEG}_{1500 \mathrm{~N}}\right)$ [23] and then purifying by dialysis. The obtained C-dots can emit green light on the UV light but the quantum yield (QY) was very lower, only 10\% after passivated by $\mathrm{PEG}_{1500 \mathrm{~N}}$. Afterwards, Hu's research group of Tianjing

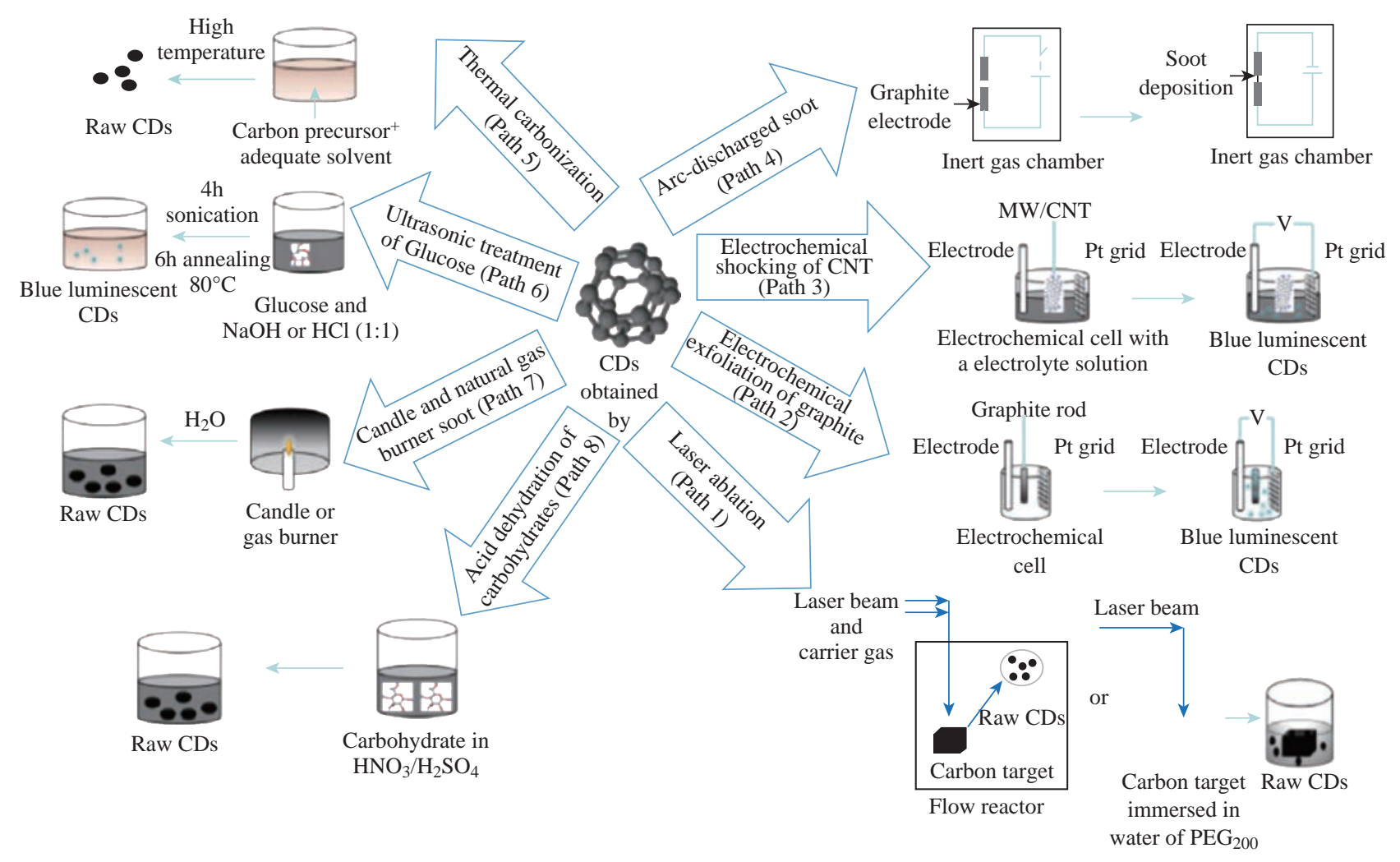

Scheme 1 Methodologies for synthesizing C-dots. Reprinted from Ref. 4 with permission. 

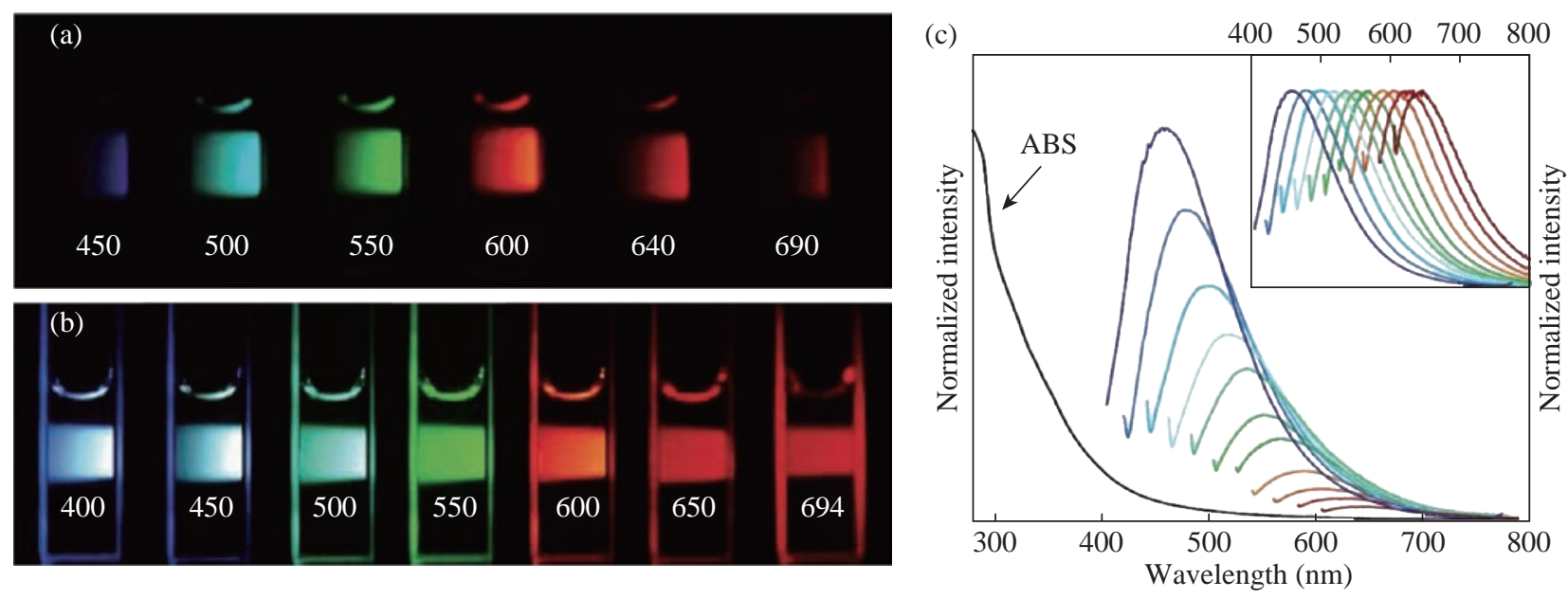

Fig. 1 (a) Aqueous solution of C-dots passivated with PEG1500N, excited at $400 \mathrm{~nm}$, and photographed through band-pass filters of different wavelengths as indicated; (b) Aqueous solution of C-dots passivated with PEG1500N, excited at the indicated wavelengths, and photographed directly; (c) Absorbance and luminescence spectra of PPEI-EI C-dots in aqueous solution formed from laser ablation methods. Reprinted from Ref. [23, 24] with permission.

University have developed a single-step procedure that integrated synthesis and passivation to get C-dots by laser irradiation of carbon powders in organic solvent [9]. And the tunable light emission of C-dots could be generated by selecting different organic solvents and modifying regents in Fig. 1. The QY of C-dots synthesized in diethanolamine is higher, reach to $11.62 \%$. Moreover, the molecular weight of solvent shrew a distinct effect on the PL peak, which shifts to a longer wavelength with the increase in the molecular weight of PEG. So they concluded that the origin of the luminescence was attributed to carboxylate ligands on the surface of the CNPs.

Electrochemical synthesis of C-dots was first demonstrated by Zheng's group of the University of Western Ontario, London [10]. They used multiwalled carbon nanotubes (MCNTs) coated on carbon paper as the working electrode to prepare C-dots by Cyclic Voltammetry. The C-dots produced were spherical, ranged from $0.5 \mathrm{~nm}$ to $2.8 \mathrm{~nm}$ in size, had lattice spacings consistent with nanocrystalline graphite, and exhibited lex-dependent PL. Subsequently, Zhou's group of Wuhan University, China produced C-dots by electro-oxidation of graphite in $0.1 \mathrm{M} \mathrm{NaH}_{2} \mathrm{PO}_{4}$ aqueous solution [11]. The two-size C-dots were obtained using centrifugal filter devices. And the PL of these C-dots was size-dependent, with emission maxima of $445 \mathrm{~nm}$ and $510 \mathrm{~nm}$ for the 1.9 and $3.2 \mathrm{~nm}$ dots, respectively. At the same time, we also confirmed that the C-dots prepared was low cytotoxicity, which are promising in biological labeling, imaging and disease diagnosis.
Compared with "top-down” approaches, "bottomup” approaches were more convenient and easy to realize large-scale to create C-dots. So many routes can be used to prepare C-dots. Among them, the most widely used are the hydrothermal method and microwave-assisted pyrolysis method, which can be realized by the one-step method for preparing fluorescent C-dots. Liu and co-workers first presenting this intriguing approach, collected candle soot as carbon precursor, oxidize in $5 \mathrm{M} \mathrm{HNO}_{3}$ and centrifugation or dialysis to collect C-dots [25]. They found that no external surface passivation agent, as required for other approaches, was needed for PL to occur by using this approach. Afterwards, Tian and co-workers purified C-dots from the combustion soot of natural gas using near same method [26]. The results of HRTEM measurements 13C NMR and FTIR revealed the C-dots were most likely to consist of a nanocrystalline core featuring graphitic sp2 carbon atoms and a surface functionalized with carboxylic/ carbonyl moieties. However, in contrast to C-dots made from candle combustion, no $\mathrm{N}$ was found to be present in these C-dots.

A facile microwave pyrolysis approach to synthesize C-dots was carried out by combining PEG200 and a saccharine (for example, glucose, fructose) in water to form a transparent solution, followed by heating in a $500 \mathrm{~W}$ microwave oven for $2-10 \mathrm{~min}$ [19]. The solution changed from colorless to tan to dark brown over the time course of the reaction. The recovered C-dots exhibited sizes and PL properties related to the duration of the microwave heating. Longer 

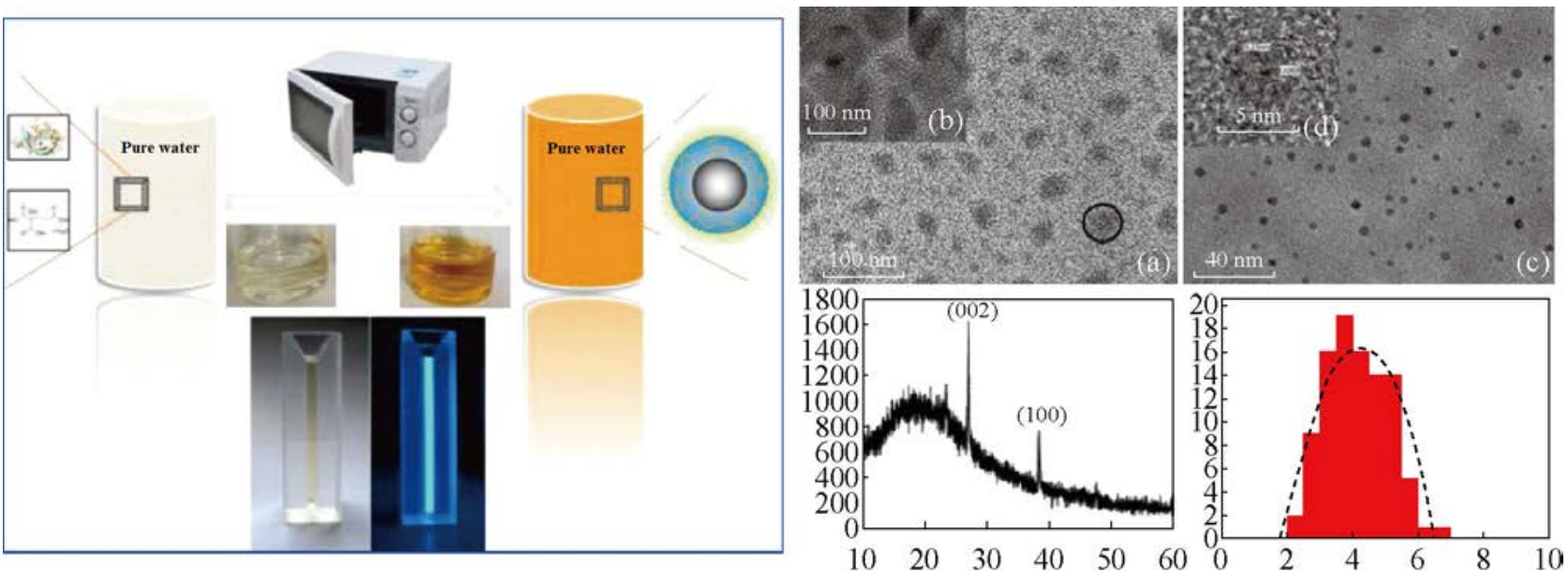

Fig. 2 (Left) Scheme of Microwave approach to C-dot Synthesis. (Right) TEM and HR-TEM images, XRD pattern, and size distribution of RNase A@C-dots. (a) TEM image of the as-prepared RNase A@C-dots inside the dialysis membrane after dialyzing against pure water. One typical RNase A@C-dot cluster is labeled with a black circle; (b) High-resolution TEM (HR-TEM) image of one focused area within the black circle; (c) TEM image of the C-dots outside the dialysis membrane; (d) HR-TEM image of one single C-dot; (e) XRD pattern of RNase A@C-dots; (f) Size distribution of C-dots. Reprinted from Ref. [21] with permission.

heating times result in the C-dots enlarging slightly and emitting at longer wavelengths. For example, the average C-dot diameters were (2.75 0.45) nm and (3.65 0.6) $\mathrm{nm}$ for heating times of 5and 10 minutes, respectively. When PEG was omitted, a similar color change was observed during microwave heating, but it is noteworthy that the non-passivated particles expressed weak and irregular PL [19].

In our group, Liu, et al. reported to synthesize fluorescent carbon dots (C-dots) with high quantum yields by using Ribonuclease A (RNaseA) as a stabilizer under microwave irradiation [21]. The preparation process and size, chemical and physical properties of C-dots have been shown in Fig. 2. The synthesized C-dots exhibited quantum yields of 24.20\%. The fluorescent color of the RNase A@C-dots can easily be adjusted by varying the microwave reaction time and microwave power. Moreover, the emission wavelength and intensity of RNase A@ C-dots displayed a marked excitation wavelengthdependent character. Notably, the PL intensity is gradually decreasing as the $\mathrm{pH}$ increases, almost linearly dependent, and the as-prepared RNase A@ C-dots are suitable for simultaneous therapy and in vivo fluorescence imaging of nude mice loaded with gastric cancer or other tumors [21].

\section{Basic Chemical and Physical Properties of C-Dots}

\section{Chemical composites}

$\mathrm{C}, \mathrm{H}$, and $\mathrm{O}$ are three fundamental elements of C-dots, but the ratios of the three elements are variable in C-dots prepared by different methods. The introduction of heteroatoms has been studied to tune the conduction/valence band position of C-dots, which confers additional functions [27-29]. For example, the quantum yield (QY) of C-dots could be significantly improved by incorporating the $\mathrm{N}$ element while C-dots with a wide band gap could be synthesized by incorporating the S element [30-34]. Furthermore, P-doped C-dots showed green fluorescence (FL), which could be utilized as an imaging signal with low background [35-37]. Moreover, other heteroatoms such as $\mathrm{Cl}$ and B could also be doped into C-dots by changing the synthetic methods or raw materials [38, 39].

\section{Optical properties}

The representative spectra of C-dots typically show a strong optical absorption in the UV region, with extending into the visible range. For example, C-dots produced from graphite by a one-step laser ablation have an excitation edge at $280 \mathrm{~nm}(4.4 \mathrm{eV})$ [9]. C-dots (2.8 $0.5 \mathrm{~nm}$ ) derived from MWCNTs by the electrooxidation method show an absorption band at $270 \mathrm{~nm}$, with a narrow full width at half maximum (FWHM) of $50 \mathrm{~nm}$ [11]. This is similar to microwave assistant produced C-dots ( $3 \mathrm{~nm}$ ) which had an absorption band at $280 \mathrm{~nm}$, also with a FWHM of $50 \mathrm{~nm}$. After surface passivation with organic regents (such as PEG200, TTDDA), C-dots absorbance was sometimes found to increase in the 350 to $550 \mathrm{~nm}$ range [19]. 
Photoluminescence (PL) of C-dots show excitation dependent emissions with few exceptions [2]. In addition, the C-dots size, $\mathrm{pH}$, and surface functional groups have significant influence on the PL of C-dots. Compared with conventional QDs, carbon-based fluorescent materials are superior in chemical stability, biocompatibility, high photostability in many studies. A recent laser scanning confocal microscopy study showed that neither blinking nor meaningful reduction in the PL intensity were observed during several hours of continuous exposure to excitation. luminescence decays further confirmed C-dots can be used in vitro and in vivo living cell or animal imaging [30].

\section{Two-photon excitation}

C-dots exhibited strong PL upon two-photon excitation in the NIR region, with an estimated twophoton absorption cross-sections comparable to those of available high performance semiconductor QDs [2, 3 , 37]. The two-photon experiments were conducted on C-dots deposited on glass substrates. A quadratic relationship between the excitation laser power and the C-dot PL intensity was found by using a femtosecond pulsed $\mathrm{Ti}$ : sapphire laser at $800 \mathrm{~nm}$ at different powers, thus confirming the two-photon excitation of the C-dots. The two-photon absorption cross-section was determined to be (39000 5000) GM (GoeppertMayer unit; $1 \mathrm{GM}=10^{-50} \mathrm{~cm}^{4}$ sphoton $^{-1}$ ) at $800 \mathrm{~nm}$ excitation. This value falls between that reported for CdSe quantum dots (780 10,300 GM) [40] and CdSe/ ZnS core-shell quantum dots $(\approx 50,000 \mathrm{GM})$ [41] for the same excitation wavelength. The emission profile of C-dots excited by two-photon has a band width comparable to the one-photon spectrum of C-dots on glass substrates, but is considerably narrower than the one photon emission spectra of C-dots in aqueous solution. This observation indicates that immobilization may influence the emission properties of the C-dots. Doped C-dots, such as $\mathrm{TiO}_{2} / \mathrm{C}$-dots, $\mathrm{SiO}_{2} / \mathrm{C}$-dots, $\mathrm{ZnO} / \mathrm{C}$-dots, and $\mathrm{ZnS} / \mathrm{C}$-dots have also shown multiphoton excitation properties as C-dots. The two-photon excitation or up-conversion process of C-dots or doped C-dots was useful for cellular imaging using twophoton luminescence microscopy or NIR imaging.

\section{Electrochemiluminescence (ECL)}

Similar to semi-QDs, C-dots exhibit ECL properties. The ECL emission of C-dots (ca. $2.0 \mathrm{~nm}$ ) produced from the electrochemical oxidation of graphite was observed as the potential was cycled between +1.8 and $-1.5 \mathrm{~V}$ (Fig. 3(a)) [13]. More recently, dual ECL peaks have been reported for a C-dot in ethanol solution containing $0.1 \mathrm{M}$ tetrabutyl ammonium bromide (TBAB) [13]. Peak 1 is attributed to the electrochemical injection of electrons in the conduction band of C-dot followed by their recombination with holes in the valence band to produce luminescence. Peak 2 is produced by the ion-ion annihilation reactions of C-dots and TBAB. Peak 1 is more sensitive to the surrounding environment in comparison with peak 2, making the latter a suited internal standard for sensing applications.

The suggested ECL mechanism involves the formation of excited-state $\mathrm{C}$-dots $\left(\mathrm{R}^{*}\right)$ by electrontransfer annihilation of negatively charged ( $\mathrm{RC}^{-}$) and positively charged $\left(\mathrm{RC}^{+}\right)$species (ET1 route in Fig. $3(\mathrm{~b})) . \mathrm{RC}^{+}$is the more stable of the two species, as indicated by the greater intensity of the cathodic ECL (Fig. 3(b)). Interestingly, when produced by microwave synthesis, $3 \mathrm{~nm}$ PEG200-functionalized C-dots also exhibited ECL behavior, but the RC' species was found to be more stable in this case [13]. The presence of

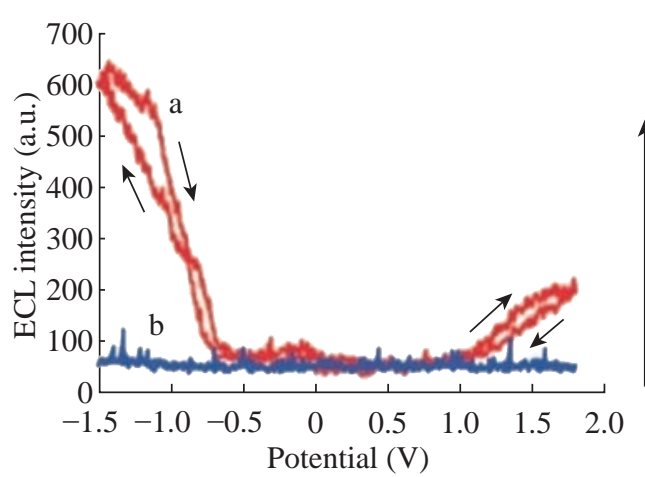

(a)

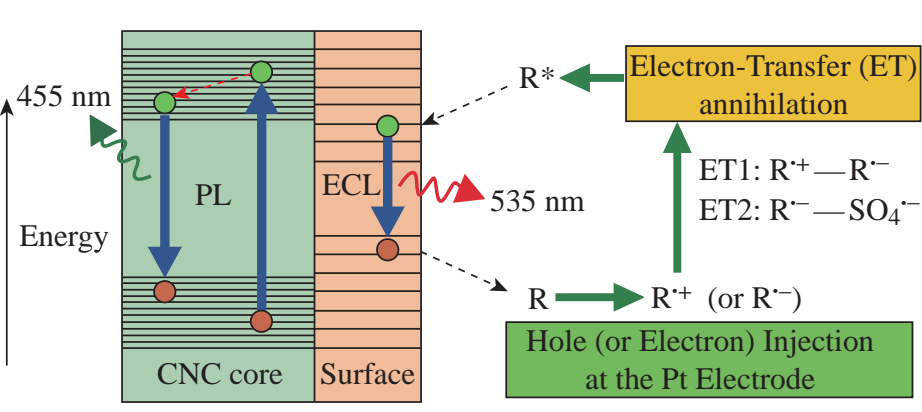

(b)

Fig. 3 (a) ECL responses with and without C-dots at a Pt electrode in $0.1 \mathrm{~m}$ phosphate buffer solution (pH 7.0) with a scan rate of 0.1 $\mathrm{V} \mathrm{s}^{-1}$; (b) The ECL and PL mechanisms in C-dots. Reproduced from Ref. [13] with permission. 
peroxydisulfate $\left(\mathrm{S}_{2} \mathrm{O}_{8}{ }^{2-}\right)$, enhanced the ECL (ET2 route in Fig. 4) in the cathodic potential range and produced a stable and sensitive (as no other coreactants tested elicited an ECL enhancement) ECL response, thus suggesting the application of C-dots for ECL sensing [19].

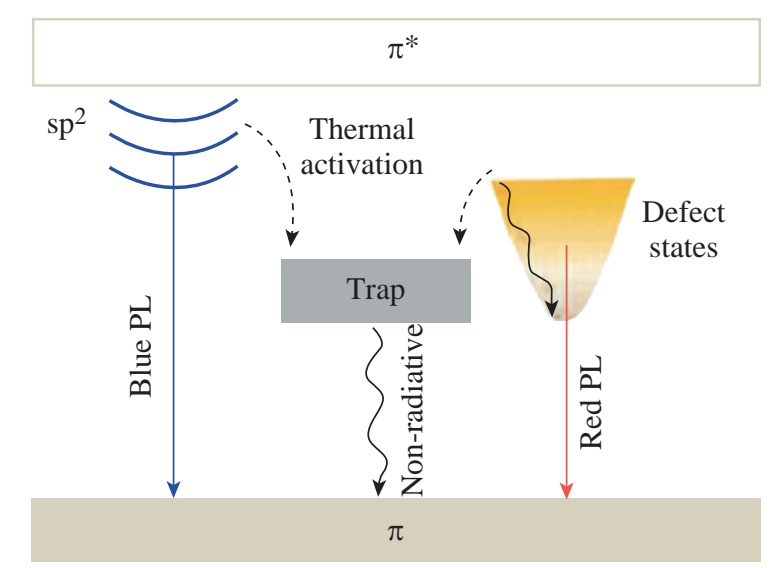

Fig. 4 Energy level structures to explain the optical behaviors of photoexcited electrons in Graphene C-dots. Reproduced with permission of American Chemical Society from Ref. [19].

\section{PL Mechanism of C-Dots}

Although the origin of their PL is a matter of debate and requires further clarification, it is highly important to explore the PL mechanism of C-dots for further guiding the development of effective synthesis routes and novel applications. Till now, four reasonable PL mechanisms have been proposed: the quantum confinement effect or conjugated $\pi$-domains, which are determined by the carbon core; the surface state, which is determined by hybridization of the carbon backbone and the connected chemical groups; the molecule state, which is determined solely by the fluorescent molecules connected on the surface or interior of the C-dots; and the cross link enhanced emission (CEE) effect [42-44]. Using densityfunctional theory (DFT) and time-dependent DFT calculations, Ding, et al. demonstrated that PL of a grapnene C-dots essentially originated from the quantum confinement of conjugated $\pi$ - electrons in sp2 carbon network and can be sensitively tuned by its size, edge configuration, shape, attached chemical functionalities, heteroatom doping and defects [45]. Xu, et al. also used singleparticle spectroscopic measurements to investigate the PL behaviors of grapnene C-dots [46]. As schematically shown in Fig. 4, photoexcited electrons through the $\pi-\pi^{*}$ transitions were proposed to relax into either the sp2 energy levels or the defect states (or call the surface state), giving rise to blue or long-wavelength PL, respectively. The blue emission might bear discrete features owing to the quantum confinement effect (QCE) of electrons inside the sp2 carbon domains. The long-wavelength emission is related to the hybrid structure comprising the oxygen functional groups (at the edges and/or on the basal planes) and the graphene core. Despite noticeable differences in the size and the number of layers from particle to particle, all of the grapnene C-dots studied possessed almost the same spectral line shapes and peak positions. This suggests that the PL of these grapnene C-dots was caused by their surface state.

Sun, et al. attribute the PL to the presence of surface energy traps that become emissive upon surface passivation [23]. They concluded that there must be a quantum confinement of emissive energy traps to the particle surface for the particle to exhibit strong PL upon surface passivation. A similar effect is seen for Silicon crystals, for which a widely accepted mechanism for luminescence emission is the radioactive recombination of exactions [45]. When coated with $\mathrm{ZnO}$ or $\mathrm{ZnS}$, the C-dots produced by Sun, et al. still required further passivation by $P G_{1500 N}$ for PL to occur [24]. All similar work proved that the surface state (or edge state) was the key PL mechanism in these types of Graphene C-dots.

\section{In vitro and In vivo Toxicity}

The toxicity of C-dots is a natural concern because of their potential for bioimaging and nanoscale dimensions. Toxicity studies have been conducted by various research groups. While the reports are few at the moment, C-dots appear to have low toxicity [2, 5, 20-25]. Ray, et al. performed cell viability tests on HepG2 cells, a human hepatocellular liver carcinomaline, using MTT and Trypan blue assays [47]. The cells were exposed to $0.1 \sim 1 \mathrm{mg} / \mathrm{L}^{-1}$ of C-dots, $2 \sim 6 \mathrm{~nm}$ in size extracted from candle soot, for $24 \mathrm{~h}$. The cell survival rate was then determined by absorbance at $550 \mathrm{~nm}$ by using the MTT assay or cell staining/counting methods for the Trypan Blue assay. The cell survival rate for a C-dot exposure of less than $0.5 \mathrm{mg} / \mathrm{mL}^{-1}$ ranged between 90 and $100 \%$. At C-dot concentrations above $0.5 \mathrm{mg} / \mathrm{mL}^{-1}$, the survival rate drops to about $75 \%$; however, the highest levels investigated were 102 to 103 times higher than necessary for bioimaging studies, thus suggesting that C-dots pose minimal toxicity effects at useful 
concentrations for bioimaging.

Cui's group have systematically investigated that the effect of C-dots on the BALB/c mice immune system $[48,49]$. In this work, mice were respectively treated with different doses of carbon dots and saline. They confirmed that high dose of C-dots could promote the percentages of $\mathrm{CD}^{3+}$ and interferon- $\gamma$ (IFN- $\gamma$ ) secretion and decrease the proportions of $\mathrm{CD}^{4+} /$ $\mathrm{CD}^{8+}$ on the firstday after administration. At 9 days post exposure, the proliferation of splenocytes had a significant increase. IFN- $\gamma$ secretion and proportions of $\mathrm{CD}^{3+} / \mathrm{CD}^{19+}$ were also found to have an obvious promotion, and both the percentages of $\mathrm{CD}^{4+}$ and $\mathrm{CD}^{8+}$ T-lymphocytes were raised, whereas the expression of cytokines made little change in the treated groups, except for IL-12 which had a slight increase in the 50 $\mathrm{mg} / \mathrm{kg}$ group. The weight coefficients and histological analysis of the spleen and thymus of the treated mice exerted fewer differences compared with those from the control mice. It suggests that carbon dots could influence the immune functions of normal BALB/C mice by inducing Th1 and Tc responses and that these effects were not enough to induce the morphological change of the immune organs.

Additionally, Yang and co-workers performed in vivo studies using CD-1 mice [50]. The mice, divided into three groups, were exposed intravenously to either 8 or $40 \mathrm{mg}$ of C-dots (ca. $5 \mathrm{~nm}, \mathrm{PEG}_{1500 \mathrm{~N}}$ passivated) or $0.9 \% \mathrm{NaCl}$ aqueous solution (nontoxic control). At 1 , 7 , and 28 days post exposure, mice were sacrificed and blood and organ samples taken for toxicological assays. During the 4 week period, no mice exposed to C-dots exhibited any adverse clinical signs or abnormal food intake. Hepatic indicators, kidney function, uric acid, blood urea nitrogen, and creatinine were all at similar levels for mice exposed to different dosages of C-dots and the $\mathrm{NaCl}$ control, thus suggesting the nontoxicity of C-dots at exposure levels and times beyond those typically used for optical in vivo imaging studies. Harvested organs also exhibited no abnormalities or necrosis. While the amount of C-dots found in the liver and spleen were higher than those found in other organs, the accumulations were relatively minor and no organ damage was present.

All the evidence points to the great potential of C-dots for in vitro and in vivo imaging studies. Although more toxicity studies need to be carried out measurements, some researchers predict that the biocompatibility of C-dots will be similar to that of current FDA-approved dyes used as optical imaging agents such as indocyanine green (LD50 = $60 \mathrm{mg} \mathrm{kg}^{-1}$ body weight) [50].

\section{Applications in Biological Medicine}

\section{Bioimaging}

Due to more biocompatible and negligible cytotoxicity, therefore not surprisingly, C-dots form an attractive alternative for bioimaging applications. The possibility of using C-dots as fluorescent labels for cellular imaging was first demonstrated by Sun, et al. who used $\mathrm{PEG}_{1500 \mathrm{~N}}$ passivated C-dots to nonspecifically stain Caco-2cells [23], as shown in Fig. 5. Soon afterward, they developed multiphoton bioimaging in living cells by using PPEI-EI-passivated C-dots [40] Their pioneering work initiated the upsurge of bioimaging with C-dots. As mentioned above, our group used Rnase $\mathrm{A}$ and amino acids to synthesize highly fluorescent C-dots, which also could be used to label cells. Notably, we first confirmed the C-dots prepared using Rnase A as template partiallocated inside the nucleus by $3 \mathrm{D}$ confocal imaging and transmission electron microscopy technologies [21].
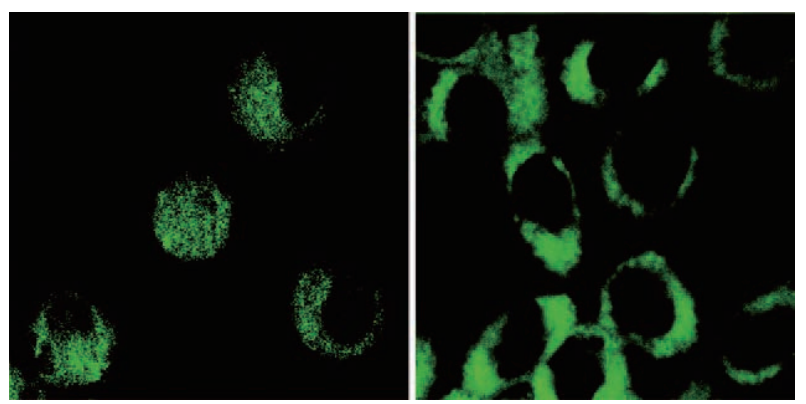

Fig. 5 Two-photon luminescence image of human breast cancer MCF-7 cells with internalized C-dots passivated with PPEI-EI. Reprinted from Ref. [40] with permission.

Ray, et al. also reported C-dots produced by burning a candle, chemically oxidizing the soot [47], for conventional bioimaging by incubating $\mathrm{a} \approx 107$ cell $\mathrm{mL}^{-1}$ solution of Ehrlich ascites carcinoma cells (EACs) with an aqueous solution of C-dots for 30 minutes. The labeled cells were imaged under an Olympus IX71 fluorescence microscope equipped with a digital camera. They demonstrated that the ability of C-dots without any further surface passivation can penetrate into the cells. Liu, et al. also found $1.5 \sim 2 \mathrm{~nm}$ C-dots uptake into E. coli and murine P19 progenitor cells and could be further imaged using laser scanning confocal 
microscopy. The PL of the C-dot could be excited over a broad range of $458 \sim 514 \mathrm{~nm}$, and showed high photostability, no blinking, and showed high photostability, no blinking, and low photobleaching.

In vivo studies of optical imaging using C-dots (ca. $5 \mathrm{~nm}$ ) produced by laser ablation have also been demonstrated [50]. In vivo fluorescent contrast agents are ideally bright, nontoxic, biocompatible, and stable against photobleaching. Both C-dots and ZnS-doped C-dots functionalized with $\mathrm{PEG}_{1500 \mathrm{~N}}$ were injected by three different routes (subcutaneously, intradermally, and intravenously) into female DBA/1 mice. Both types of C-dots could be excited through $470 \mathrm{~nm}$ and $545 \mathrm{~nm}$ filters. The ZnS-doped C-dots emitted more strongly than nondoped C-dots, which is consistent with their PL behavior in solution. The PL from both types of injected dots faded $24 \mathrm{~h}$ after injection. After intradermal injection into thefront extremity, the C-dots migrated to the axillary lymphnodes, similar to CdSe/
ZnS semiconductor quantum dots, butat a slower rate, presumably because of PEG functionalization reducing their interaction with lymph cells. When injected intravenously for whole-body circulation studies (Fig. 6), C-dot emission from the bladder area was observed, and $3 \mathrm{~h}$ after injection PL could be detected in the urine. At $4 \mathrm{~h}$ after injection, organs were harvested and the C-dots were found to have accumulated in the kidney(which is consistent with a urine excretion pathway) and scantly in the liver. Although significant hepatic uptake is known for nanoparticles and nanotubes, the lower levels observed for C-dots was attributed to the surface PEG likely reducing their protein affinity [40].

\section{Drug/Gene delivery}

In the other research, Tang, et al. have developed a FRET-based C-dots drug delivery platform for realtime monitoring and tracking of the release of the
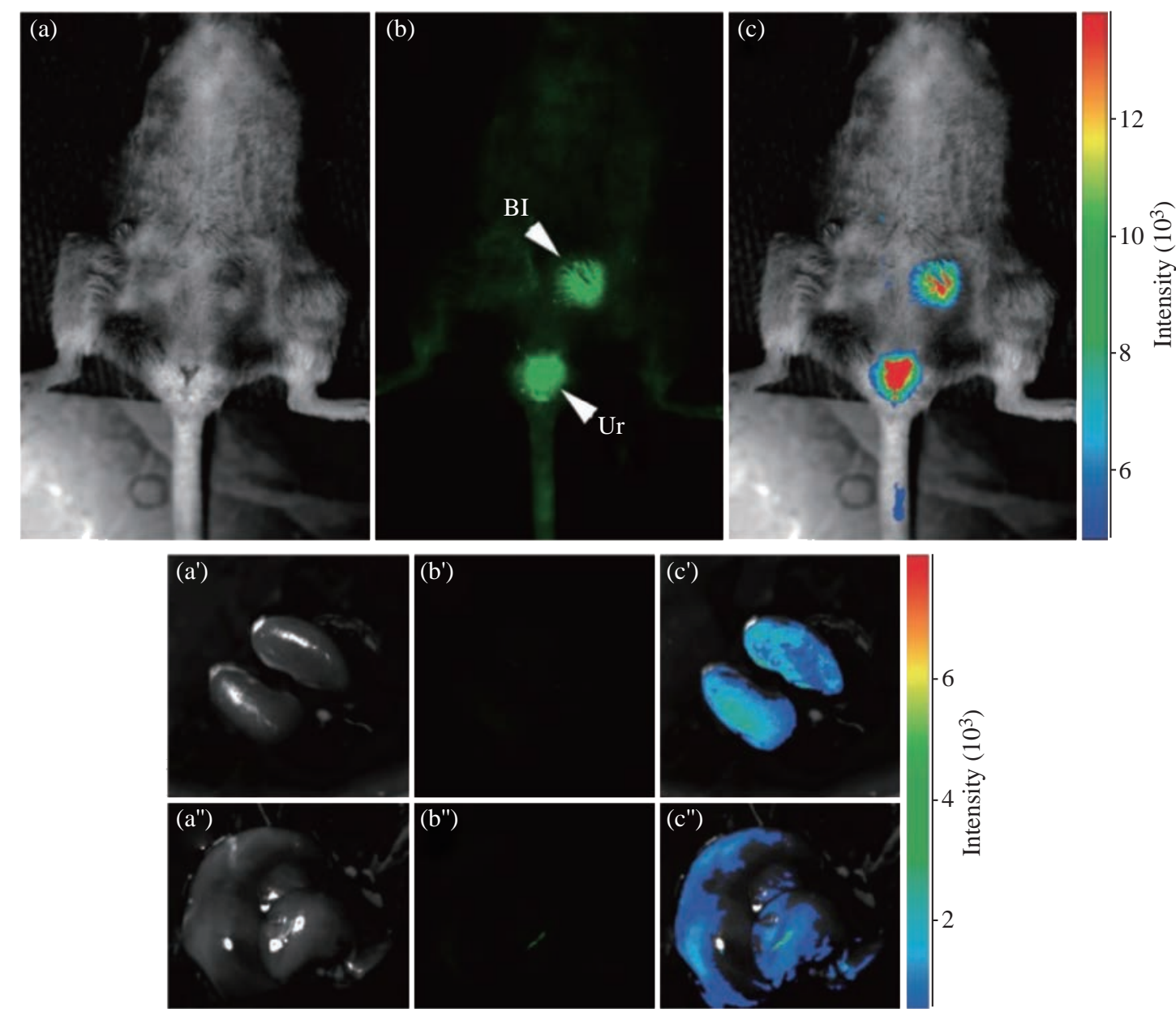

Fig. 6 Intravenous injection of C-dots: (a) Bright field; (b) As-detected fluorescence (Bl, bladder; Ur, urine); and (c) Color-coded images. The same order is used for the images of the dissected kidneys $\left(\mathrm{a}^{\prime}-\mathrm{c}^{\prime}\right)$ and liver $\left(\mathrm{a}^{\prime \prime}-\mathrm{c}^{\prime \prime}\right)$. Reprinted from Ref. [50] with permission. 


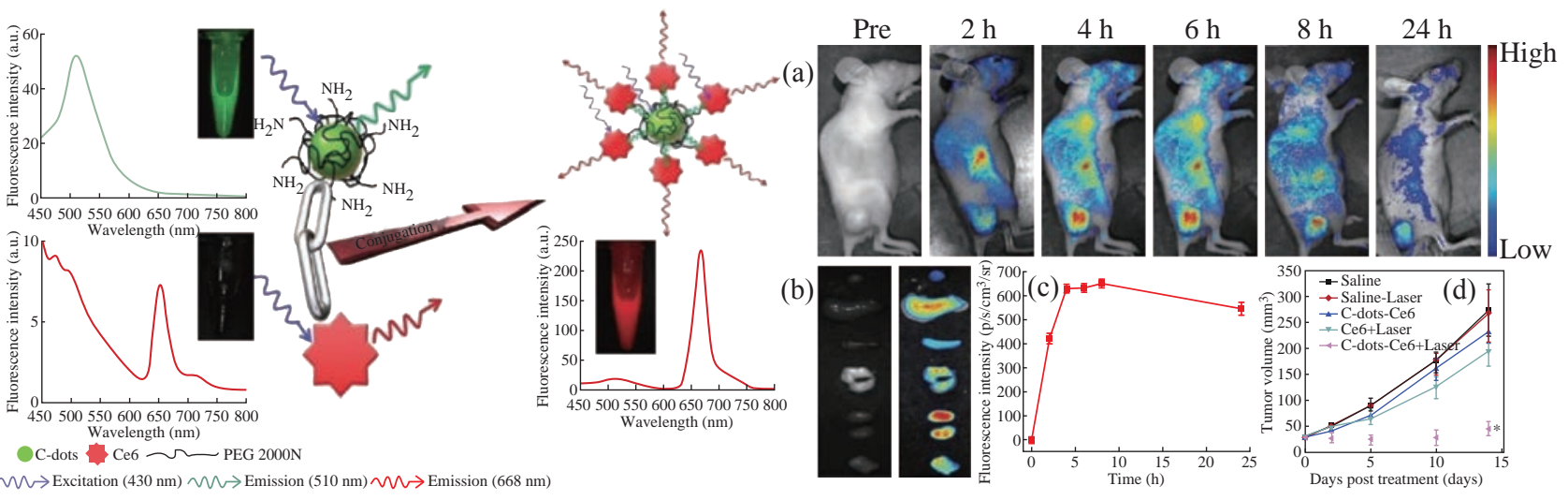

Fig.7 (Left) Scheme of Förster (fluorescence) resonance energy transfer (FRET) process between C-dots and Ce6. (Right) (a) Realtime in vivo NIR fluorescence images after intravenous injection of C-dots-Ce6 in nude mice at different time points; (b) Ex vivo images of mice tissues (from top to bottom: heart, liver, spleen, lung, kidneys, tumor); (c) the average fluorescence intensities from the tumor are at 24 h post-injection $(n=5)$; (d) MGC803 tumor growth curves after various treatments $(n=5)$. ( $*, P<0.05$ for other groups versus C-dots-Ce6 laser group). Reprinted from Ref. [52] with permission.

fluorescent drug molecule [51]. C-dots offer excellent biocompatibility, stable fluorescence, and efficient FRET between C-dots and the attached fluorescent drug molecules, such as FRET-Cdot-DDS for twophoton imaging and monitoring of drug release in tumor tissues with the thickness of $65 \sim 300 \mu \mathrm{m}$ have been demonstrated. They also predicted that further development of synthesis and surfacechemistry-engineered doxorubicin, enabling enhanced drug delivery, convenient cell imaging, and realtime monitoring of drug release. Moreover, the capabilities of using C-dots for FRET-based imaging and targeted delivery modalities will allow more new exciting opportunities for cancer diagnosis and treatment, showing improved imaging sensitivity and therapeutic efficacy.

Our group has designed and developed a novel theranostic platform based on photosensitizerconjugated C-dots is successfully [52]. C-dots-Ce6 is a good candidate with excellent imaging and tumor-homing ability fo rNIR fluorescence imaging guided PDT treatment. The prepared C-dots-Ce6 owns good stability, high water dispersibility and solubility, noncytotoxicity, good biocompatibility, enhanced photosensitizer fluorescence detection and remarkable photodynamic efficacy upon irradiation9 (Fig. 7). Our results indicated that the synthesized multifunctional nanocarrier platform is effective for simultaneous enhanced-PFD and PDT of gastric cancer tumor in vivo.

Tiwari, et al. explored the drug delivery capacity of C-dots for targeted delivery of doxorubicin (DOX) [54]. they have used folic acid (FA) as a targeted molecule due to its high expression in most cancer cells. DOX was successfully anchored and the drug loading capacity was calculated to be very high $(\sim 86 \%)$ thus improving the efficiency of C-dots as drug delivery vehicles.The DOX@C-dots conjugate showed an ideal drug release profile at physiological as well as slightly acidic $\mathrm{pH}$ and was found to follow first order release kinetics based on the statistical calculations. C-dots were highly biocompatible with both the cells tested.DOX@C-dots showed a higher killing rate of cancer cells than free DOX and was found to be less toxic to normal cells due to FA mediated targeting. C-dots prepared by microwave assisted synthesis using gum arabic (GA), and its use as molecular vehicle to ferry ciprofloxacin hydrochloride, a broad spectrum antibiotic, was reported by Thakur, et al. [55]. Ciprofloxacin can be easily anchored to selffunctionalized C-dots without involvement of stringent protocols. Loading capacity of C-dots (> 90\%) shows it as an ideal vehicle for ferrying significant amount of clinical pay loads. Also, path of C-dots can be traced due to its magnificent photoluminescence properties. The conjugate was a potent antimicrobial in nature against both gram positive and gram negative bacteria. Potential antibiotics like ciprofloxacin can be released at sustained rate from the surface of C-dots, following Higuchi model under physiological conditions.

In 2012, Liu, et al. demonstrated that gene delivery using PEI-functionalized C-dots (CD-PEI) as gene delivery carriers was successful [56]. The positively charged CD-PEI is able to mediate 
transfection of DNA molecules in COS-7 and HepG2 cells with higher efficiency and lower cytotoxicity as compared with the polymer control (PEI25k). The multicolor fluorescence of the C-dot/DNA complexes could be clearly observed in cytosol, demonstrating the great potential of CD-PEI in bioimaging and biolabeling. The DNA transfection was lightened by C-dots under confocal microscopy. Lee, et al. also demonstrated real time monitoring of gene delivery by combining $\mathrm{C}$-dots with metal nanoparticles [57]. Blue-emitting CD-PEI and PEI functionalized Au-nanoparticles (Au-PEI) were first complexed with TOTO-iodide (red emission) labeled plasmid DNA(pDNA) via electrostatic interaction, leading to initial fl uorescence quenching. The complex of CDPEI/pDNA/Au-PEI was up-taken into the cytosol as shown by co-localization of pDNAsignal and moderately quenched CD-PEI signal. Gene dissociation induced by low-pH inside cancer cells was evidenced by the recovery of CD-PEI blue fluorescence localized in thecytosol and pDNA fluorescence localized in the nucleus.

Our group has also developed a microwave assisted one-step synthesis of C-dots with citric acid as carbon source and tryptophan (Trp) as both nitrogen source and passivation agent [58]. The C-dots are uniform in size and show superior water solubility, excellent biocompatibility, and high quantum yield. Furthermore, PEI (polyethylenimine)-adsorbed C-dots nanoparticles (C-dots@PEI) were developed to deliver Survivin siRNA into human gastric cancer cells MGC803. The resulting nanocarrier exhibited excellent biocompatibility and a significant increase in cellular delivery of siRNA was observed, inducing efficient knockdown for Survivin protein to 6.1\%. In addition, C-dots@PEI complexes mediated Survivin silencing, the arrested cell cycle progression in $G$ phase as well as cell apoptosis was observed. The results indicate that C-dots-based nanocarriers can lead to a broad range of siRNA delivery systems for cancer therapy.

\section{Biosensor}

In this case, through interaction with the functional groups on the C-dots, the target ions can easily effectively quench the FL of C-dots through an electron or energy transfer process. For example, Zhou, et al. have demonstrated a type of C-dots-based sensor for label-free detection of $\mathrm{Hg}^{2+}$ and biothiols with high sensitivity and selectivity [59]. $\mathrm{Hg}^{2+}$ can quench the FL of the C-dots while biothiols can effectively shelter the
FL quenching due to their ability to remove $\mathrm{Hg}^{2+}$ from the surface of C-dots. Zhao, et al. reported that they prepared C-dots by a facile, economic and green onestep hydrothermal synthesis route using dopamine as source. The as-prepared C-dots have an average size about $3.8 \mathrm{~nm}$ [60]. The emission spectra of the C-dots are broad, ranging from approximately 380 (purple) to approximately $525 \mathrm{~nm}$ (green), depending on the excitation wavelengths. Due to the special response of catechol groups to $\mathrm{Fe}^{3+}$ ions, the C-dots can serve as a very effective fluorescent sensing platform for labelfree sensitive and selective detection of $\mathrm{Fe}^{3+}$ ions and dopamine with a detection limit as low as $0.32 \mathrm{~mm}$ and $68 \mathrm{~nm}$, respectively. The new "mix-and-detect" strategy is simple, green, and exhibits high sensitivity and selectivity. The present method was also applied to the determination of $\mathrm{Fe}^{3+}$ ions in real water samples and dopamine in human urine and serum samples successfully. Many other raw materials were chosen to synthesize C-dots that could be used to detect ions such as $\mathrm{Cu}^{2+}, \mathrm{Hg}^{2+}, \mathrm{Ag}^{+}, \mathrm{Cr}^{3+}, \mathrm{Al}^{3+}$, and $\mathrm{H}^{+}$directly [6164].

In 2011, researchers first achieved the detection of DNA; Bai and other studies [65] have found that methylene blue can effectively quench the fluorescence of C-dots; then they joined ct-DNA in solution; the fluorescence of the solution was found to be restored. Based on this phenomenon, a new fluorescent sensor for DNA detection was designed; the detection limit was $1.0 \times 10^{-6} \mathrm{~mol} / \mathrm{L}$ with the linear ranging from $3.0 \times 10^{-6} \mathrm{~mol} / \mathrm{L}$ to $8.0 \times 10^{-5} \mathrm{~mol} / \mathrm{L}$. The C-dot-based sensor was also used to detect micro RNA124a expression during neurogenesis [66]. The C-dots derived from candle soot were conjugated with double-stranded DNA (dsDNA) beacon contained a micro RNA124a-binding oligonucleotide and a microRNA124a-sensing oligonucleotide (a black hole quencher on its terminal that could quench the FL of C-dots). Then themodified C-dots were incubated with P19 cells to sense microRNA124a expression. The microRNA124a, which is highly expressed during neurogenesis, was bound to the microRNA124abinding oligonucleotide, resulting in the separation of the sensing oligonucleotide from the C-dots and, in consequence, the recovery of PL.

\section{Summy and Outlook}

In this review, we have briefly introduced the synthesis methods, properties and application in 
biomedicine engineering, and we have described recent advances in elucidating the PL mechanism of these materials. A wide range of approaches (top-down cutting and bottom-up carbonization) already exist for producing C-dots with controllable characteristics. Investigation of the PL properties of C-dots includes the different emission centers, excitation dependence, and $\mathrm{pH}$ and solvent sensitivity, all of which are hot research topics. Although a variety of C-dots with different chemical structures have been developed, four probable PL principles have been elucidated: the quantum confinement effect or conjugated $\pi$-domains, which are determined by the carbon core; the surface state, which is determined by hybridization of the carbon backbone and connected chemical groups; the molecule state, which is determined solely by the fluorescent molecules connected on the surface orinterior of the C-dots; and the crosslink enhanced emission (CEE) effect.

The emergence of C-dots opens a brand new chapter in bioimaging and biosensing. As a promising substitute for semiconductor QDs, C-dots have been widely used in biology due to their biocompatibility and low toxicity. Different synthetic methods, starting materials, and surface modification processes can generate a wide variety of C-dots, which cannot only find application in bioimaging and biosensing both in vitro and in vivo but also serve as smart drug delivery vehicles.

Nonetheless, some problems still exist that impede the further bio-applications of C-dots: (1) It is difficult to synthesize C-dots with uniform size. Proverbially, the uniformity has a great influence on the properties of C-dots, which further disturb the bioapplications of C-dots. Consequently, almost all of the synthesized C-dots need further separation and purification. (2) C-dots obtained from different batches often show different properties, such as size, QY, and FL. This problem limits the commercialization of C-dots, which needs to be settled urgently. (3) Some C-dots obtained at the very start are not fluorescent, which need further passivation. Furthermore, C-dots with more excellent properties should be explored for their further applications. For example, in biosensing and bioimaging, the FL of C-dots is the fundamental property that should be studied and brighter C-dots should be developed. In drug delivery, C-dots often serve as delivery vectors and provide FL signals. In the next stage, C-dots with more distinguishing FL and functional groups should be exploited. In other bioapplications, C-dots with extraordinary functional groups or modifications should be studied. Although these problems disturb the bio-applications of C-dots, the advantages of C-dots are still remarkable. They possess low toxicity, excellent water solubility, ease of synthesis and functionalization, and outstanding photostability. Much effort is worth devoting to further exploring versatile C-dots in biomedical and nano-device applications.

\section{Acknowledgements}

This work is supported by National Natural Scientific Fund (Nos. 31170961), 863 project of China (2012AA022703), and Shanghai Jiao Tong University Fund (No. YG2014MS01).

\section{References}

[1] X. Xu, R. Ray, Y. Gu, et al., Electrophoretic analysis and purification of fluorescent single-walled carbon nanotube fragments. J. Am. Chem. Soc., 2004, 126: 12736-12737.

[2] S. N. Baker, G. A. Baker, Luminescent carbon nanodots: emergent nanolights. Angew. Chem. Inter. Edt., 2010, 49: 6726-6744.

[3] C.Q. Ding, A.W. Zhu and Y. Tian, Functional surface engineering of C-dots for fluorescent biosensing and in vivo bioimaging. A. Chem. Res., 2014, 47: 20-30.

[4] D.J. Silva, H.M.R. Goncalves, Analytical and bioanalytical applications of carbon dots. Trac-Trends Anal Chem., 2011, 30: 1327-1336.

[5] H. Zhang, Y. Chen, M. Liang, et al., Solid-phase synthesis of highly fluorescent nitrogen-doped carbon dots for sensitive and selective probing ferric ions in living cells. Ana. Chem., 2014, 86(19): 9846-9852.

[6] Y.P. Sun, Y. He, H. Lu, et a1., Cytotoxicity of cadmium based, aqueous phase-synthesized, quantum dots and its modulation by surface Coating. Biomaterials, 2009, 30: 19-25.

[7] S. Hu, J. Liu, S. Cao, et a1. Laser synthesis and size tailor of carbon quantum dots. J. Nanopart. Res., 2011, 13: 7247-7252.

[8] X. Li, H. Wang, Y Shimizu., et al., Preparation of carbon quantum dots with tunable photoluminescence by rapid laser passivation in ordinary organic solvents. Chem. Commun., 2010, 47: 932-934.

[9] S.L. Hu, K.Y. Niu, J. Sun, et al., One-step synthesis of fluorescent carbon nanoparticles by laser irradiation. $J$. Mater. Chem., 2009, 19: 484-488.

[10] L.Y. Zheng; Y. Chi, W. Dong, et al., Electrochemiluminescence of water-soluble carbon nanocrystals released electrochemically from graphite. $J$. Am. Chem. Soc., 2009, 131: 4564-4565.

[11] J. Zhou, C. Booker, Z. Ding, et a1. An electrochemical avenue to blue luminescent nanocrystals from multiwalled carbon nanotubes (MWCNTs). J. Am. Chem. Soc., 2007, 129: 744-745.

[12] Q. Zhao, Z. Zhang, D. Pang, et a1. Facile preparation of low cytotoxicity fluorescent carbon nanocrystals by electro-oxidation of graphite. Chem. Commun., 2008, 
5116-5118.

[13] L. Zheng, Y. Chi, Y. Dong, et al., Electrochemilumiescence of water-soluble carbon nanocrystals released electrochemically from graphite, J. Am. Chem. Soc., 2009, 131(13): 4564-4565.

[14] Z.C. Yang, M. Wang, A. M. Yong, et al. Intrinsically fluorescent carbon dots with tunable emission derived from hydrothermal treatment of glucose in the presence of mono potassium phosphate. Chem. Commun., 2011, 47: 11615-11617.

[15] H. Peng, T.S. Jadranka, Simple aqueous solution route to luminescent carbogenic dots from carbohydrates. Chem. Mater., 2009, 21: 5563-5565.

[16] H. Li, X. He, Y. Liu, et al., One-step ultrasonic synthesis of water-soluble carbon nanoparticles with excellent photoluminescent properties. Carbon, 2011, 49: 605-609.

[17] A.B. Bourlinos, A. Stassinopoulos, D. Anglos, et al., Surface functionalized carbogenic quantum dots. Small, 2008, 4: 455-458.

[18] R.L. Liu, S.H. Wu, K.K. Liu, et al., An aqueous route to multicolor photoluminescent carbon dots using silica spheres as carriers. Angew. Chem., 2009, 121: 4668-4671.

[19] H. Zhu, X.L. Wang, Y.L. Li, et al. Microwave synthesis of fluorescent carbon nanoparticles with electrochemiluminescence properties. Chem. Commun., 2009, 34: 5118-5120.

[20] L. Cao, X. Wang, M.J.Meziani, et al., Carbon dots for multiphoton bioimaging. J. Am. Chem. Soc., 2007, 129: 11318-11319.

[21] H.Y. Liu, Q. Wang, G. Shen, et al. A mutifunctional rebonuclease A-conjugated carbon dots cluster nanosystem for synchronous cancer imaging and therapy. Nano. Res, Let., 2004, 9: 397-408.

[22] H. Zhu, X. Wang, Y. Li, et al., Microwave synthesis of fluorescent carbon nanoparticles with electrochemiluminescence properties. Chem. Commun., 2009, 5118-5120.

[23] Y.P. Sun, B. Zhou, Y. Lin, et al., Quantum-sized carbon dots for bright and colorful photoluminescence. J. Am. Chem. Soc., 2006, 128: 7756-7757.

[24] Y.P. Sun, X. Wang, F. Lu, et al., Doped carbon nanoparticles as a new platform for highly photoluminescent dots. J. Phys. Chem. C., 2008, 112: 18295-18298.

[25] H. Liu, T. Ye, C. Mao, et al., Fluorescent carbon nanoparticles derived from candle soot. Angew. Chem. Int. Ed., 2007, 46: 6473-6475.

[26] L. Tian, D. Ghosh, W. Chen, et al., Nanosized carbon particles from natural gas soot [J]. Chem. Mater., 2009, 21: 2803-2809.

[27] J. Lu, J.X. Yang, J. Wang, et al., One-pot synthesis of fluorescent carbon nanoribbons, nanoparticles, and graphene by the exfoliation of graphite in ionic liquids. ACS Nano, 2009, 3: 2367-2375.

[28] C.W. Lai, Y.H. Hsiao, Y.K. Peng, et al., Facile synthesis of highly emissive carbon dots from pyrolysis of glycerol; gram scale production of carbon dots/mSiO(2) for cell imaging and drug release. J Mater Chem., 2012, 22: 14403-14409.

[29] A.B. Bourlinos, A. Stassinopoulos, D. Anglos, et al., Photoluminescent carbogenic dots. Chem. Mater., 2008, 20: 4539-4541.

[30] W. Wei, C. Xu, L. Wu, et al., Non-enzymatic browningreaction: a versatile route for production of nitrogendoped carbon dots with tunable multicolor luminescent display. Sci Rep, 2014, 4: 35-64.

[31] Z. Qian, J. Ma, X. Shan, et al., Highly luminescent $\mathrm{N}$-doped carbon quantum dots as an effective multifunctional fluorescence sensing platform. Chemistry, 2014, 20: 2254-2263.
[32] Y. Xu, M. Wu, Y. Liu, et al., Nitrogen doped carbon dots: a facile and general preparation method, photoluminescence investigation, and imaging applications. Chemistry, 2013, 19: 2276-2283.

[33] Y. Dong, H. Pang, H.B. Yang, et al., Carbon based dots co-doped with nitrogen and sulfur for high quantum yield and excitation-independent emission. Angew Chem Int Ed Eng, 2013, 52: 7800-7804.

[34] S. Chandra, P. Patra, S.H. Pathan, et al., Luminescent S-doped carbon dots: an emergent architecture for multimodal applications. J Mater Chem B, 2013, 1: 23752382.

[35] W. Wang, Y.M. Li, L. Cheng, et al., Water-soluble and phosphorus-containing carbon dots with strong green fluorescence for cell labeling. J Mater Chem B, 2014, 2: 46-48.

[36] Z.Q. Xu, L.Y. Yang, X.Y. Fan, et al., Low temperature synthesis of highly stable phosphate functionalized two color carbon nanodots and their application in cell imaging. Carbon, 2014, 66: 351-360.

[37] S. Chandra, P. Das, S. Bag, et al. Synthesis, functionalization and bioimaging applications of highly fluorescent carbon nanoparticles. Nanoscale, 2011, 3: 1533-1540.

[38] S. Jahan, F. Mansoor, S. Naz, et al., Oxidative synthesis of highly fluorescent boron/nitrogen co-dopedcarbon nanodots enabling detection of photosensitizer and carcinogenic dye. Anal Chem, 2013, 85: 10232-10239.

[39] S. Hu, R. Tian, Y. Dong, et al., Modulation and effects of surface groups on photoluminescence and photocatalytic activity of carbon dots. Nanoscale, 2013, 5: 11665-11671.

[40] L. Cao, X. Wang, M.J. Meziani, et al., Carbon dots fot multiphoton bioimaging, J Am. Chem, Sco., 2007, 129(37): 11318-11319.

[41] S.C. Pu, M.J. Yang, C.C. Hsu, et al., The empirical correlation between size and two-photon absorption cross section of CdSe and CdTe quantum dots. Small, 2006, 2: 1308-1313.

[42] S.L.Hu, R.X.Tian, L.L.Wu, et al., Chemical regulation of carbon quantum dots from synthesis to photocatalytic activity. Chem-Asian J., 2013 , 8: 1035.

[43] S.J. Zhu, J.H. Zhang, S.J. Tang, et al., Surface chemistry routes to modulate the photoluminescence of graphene quantum dots: From fluorescence mechanism to upconversion bioimaging applications. Adv. Funct. Mater., 2012, 22: 4732-4740.

[44] L.L. Li, J. Ji, R. Fei, et al., A facile microwave avenue to electrochemiluminescent two-color graphene quantum dots. Adv. Funct. Mater, 2012, 22: 2971-2979.

[45] Z.F. Ding, B.M. Quinn, S.K. Haram, et al., Electrochemistry and electrogenerated chemiluminescence from silicon nanocrystal quantum dots. Science, 2002, 296: 1293 - 1297.

[46] Q.F. Xu, Q. Zhou, Z. Hua, et al., Single-particle spectroscopic measurements of fluorescent graphene quantum dots. ACS Nano, 2013, 7: 10654-10661.

[47] S.C. Ray, A. Saha, N.R. Jana, et al., Fluorescent carbon nanoparticles: Synthesis, characterization, and bioimaging application. J. Phys. Chem. C, 2009, 113: 18546-18551.

[48] K. Wang, Z. Gao, Y. Wang, et al., Systematic safety evaluation on photoluminescent carbon dots. Nanoscale Research Letters, 2013, 8: 122.

[49] Z. Gao, G. Shen, X. Zhao, et al., Carbon dots: A safe nanoscale substance for the immunologic system of mice. Nanoscale Research Letters, 2013, 8(278): 1-8.

[50] S.T. Yang, X. Wang, H. Wang, et al., Carbon dots as nontoxic and high-performance fluorescence imaging agents. J. Phys.Chem. C, 2009, 113: 18110-18114.

[51] J. Tang, B. Kong, H. Wu, et al., Carbon nanodots featuring efficient FRET for real-time monitoring of drug delivery 
and two-photon imaging. Adv Mater, 2013, 25: 65696574.

[52] P. Huang, J. Lin, X. Wang, et al., Light-triggered theranostics based on photosensitizer-conjugated carbon dots for simultaneous enhanced-fluorescence imaging and photodynamic therapy. Advanced Materials, 2012, 24: 5104-5110

[53] S. Pandey, M. Thakur, A. Mewada, et al., Carbon dots functionalized gold nanorod mediated delivery of doxorubicin: tri-functional nano-worms for drug delivery, photothermal therapy and bioimaging. J. Mater. Chem. B, 2013, 1: 4972-4982.

[54] A. Tiwari, V. Singh, Microwave-induced synthesis of electrical conducting gum acacia-graft-polyaniline. Carbohydrate Polymers, 2008, 74(3,4): 427-434.

[55] M. Thakur, S. Pandey, A Mewada et al., Antibiotic conjugated fluorescent carbon dots as a theranostic agent for controlled drug release, bioimaging, and enhanced antimicrobial activity. Journal of Drug Delivery, 2014, 1-9.

[56] C. Liu, P. Zhang, X. Zhai, et al., Nano-carrier for gene delivery and bioimaging based on carbon dots with PEIpassivation enhanced fluorescence. Biomaterials, 2012, 33: 3604-3613.

[57] D.E. Lee, H. Koo, I. Cheol et al., Multifunctional nanoparticles for multimodal imaging and theragnosis. Chem. Soc. Rev., 2012, 41: 2656-2672.

[58] Q. Wang, C. Zhang, G. Shen, et al., Fluorescent carbon dots as an efficent siRNA nanocarrier for its interference therapy in gastric cancer cells. J. Nanobiotech, 2014, 12: 58-73.

[59] L. Zhou, Y. Lin, Z. Huang, et al., Carbon nanodots as fluorescence probes for rapid, sensitive, and label-free detection of $\mathrm{Hg}^{2+}$ and biothiols in complex matrices. Chem. Commun., 2012, 48: 1147-1149
[60] A. Zhao, C. Zhao, M. Li, et al., Ionic liquids as precursors for highly luminescent, surface-different nitrogen-doped carbon dots used for label-free detection of $\mathrm{Cu}^{2+} / \mathrm{Fe}^{3+}$ and cell imaging. Anal Chim Acta, 2014, 809: 128-133.

[61] X. Ran, H. Sun, F. Pu, et al., Ag nanoparticledecoratedgraphene quantum dots for label-free, rapid and sensitivedetection of $\mathrm{Ag}^{+}$and biothiols. Chem Commun (Camb), 2013, 49: 1079-1081.

[62] A. Salinas-Castillo, M. Ariza-Avidad, C. Pritz, et al., Carbon dots for copper detection with down and upconversion fluorescent properties as excitation sources. Chem Commun (Camb), 2013, 49: 1103-1105.

[63] Y.Q. Dong, R.X. Wang, G.L. Li, et al., Polyamineunctionalized carbon quantum dots as fluorescent probes for selective and sensitive detection of copper ions. Anal Chem, 2012, 84: 6220-6224.

[64] L. Liu, Y. Li, L. Zhan,et al., One-step synthesis of fluorescent hydroxyls-coated carbon dots with hydrothermal reaction and its application to optical sensing of metal ions. Sci China Chem, 2011, 54: 13421347.

[65] W.J. Bai, H.Z. Zhang, X.J. Mao, et al., A carbon dots-based fluorescence turn-on method for DNA determination. Analytical Sciences, 2011, 27(3): 243-246.

[66] E.H. Noh, H.Y. Ko, C.H. Lee, et al., Carbon nanodotbased self-delivering microRNA sensor to visualize microRNA124a expression during neurogenesis. J Mater Chem B, 2013, 1: 4438-4445.

Copyright $₫ 2016$ Guangxia Shen, Meixin Hu and Jianrong Qi. This is an open-access article distributed under the terms of the Creative Commons Attribution License, which permits unrestricted use, distribution, and reproduction in any medium, provided the original author and source are credited. 